\title{
How to Understand Digital Studio Outputs: The Case of Digital Music Production
}

\author{
Karim Barkati and Francis Rousseaux \\ Institut de Recherche et Coordination Acoustique/Musique (IRCAM), France \\ karim.barkati@ircam.fr \\ Reims Champagne-Ardenne University, CReSTIC EA 3804 \& IRCAM, France
}

francis.rousseaux@univ-reims.fr

\begin{abstract}
Digital studios trace a great amount of processes and artifacts. The important flow of these traces calls for a system to support their interpretation and understanding. We present our design and development of such a system in the digital music production context, within the Gamelan research project. This trace-based system is structured in three main layers: track production process, interpret collected traces according to a dedicated domain ontology, help querying and visualizing to foster production understanding. We conclude by discussing some hypotheses about trace-based knowledge engineering and digital music production understanding.
\end{abstract}

Keywords: Digital Music Production, Digital Artifact Preservation, Trace Engineering, Process Understanding, Digital Humanities, Digital Studio.

\section{Introduction}

\section{$1.1 \quad$ Motivation and Objectives}

From a social standpoint, the large and ever growing number of users of audio environments for personal or applied production makes the music production field one of the richest in evolution. However, the complexity of the production management is a well-known effect in the community and is often described as revealing an inconsistency between the tools used. Indeed, the industry provides tools that are more and more powerful but regardless of global usage: users combine multiple tools simultaneously or constantly alternate from one tool to another.

From a legal standpoint, there is a real problem of content tracking, given their multiple uses or changes in production. Till now, audio production systems have kept no operational tracks that would allow following up the rights associated with each element.

Indeed, music tool design mainly focuses on the making of the final product, because the very first aim of the studio is to provide the creator with efficient means to make and shape the musical object they came in the studio for. But 
this requisite priority on creativity has overshadowed other needs that appear later: recovery and understanding. The Gamelan project aims at demonstrating that appropriately using knowledge management tools for trace-based systems [12, we now have both theoretical and practical means to build a system that can help understanding digital studio outputs, i.e. effective means to bring music production data and process to the Knowledge Level [3].

This paper describes how we designed and developed such a system upon a digital music production environment: the Gamelan "meta-environment". As far as we know, such a management and archiving system has not been built for music production before. It has been designed in three layers, as shown on Fig.1]

1. Track production process (software events and files),

2. Interpret collected traces according to a domain ontology (DiMPO),

3. Help querying and visualizing to foster production understanding.

These three parts set the three central paper sections - Production tracking system, Trace interpretation system, and Querying and visualizing system followed by Discussion and Conclusions.

\subsection{Use Cases and Results}

The Gamelan project embraces various creative practices related to its partners core business and expertise, who defined three main use cases.

IRCAM. Recovery assistance and synthesis of information from one phase to another of a record. Follow the recording and editing situation of the piece Nuages gris of Franz Liszt in the Liszt as a Traveler CD played by pianist Emmanuelle Swiercz. - Identify and represent the work sessions in two dimensions by time and by agent, all the events of one session (creation, update, export), and the dependencies of import and export files between sessions.

INA/GRM. Identification of files that have contributed to the final version of a work. Log every DAW operation of a composer during the composition of a jingle. - Ensure that the file called "Final-Mixdown" is actually the one that produced the latest audio files of the work; identify possible format changes (stereo, 8-channel, mp3); identify the intermediate versions; detect missing data and check information integrity are key features.

EMI Music. Recovery and edit of past productions; Contributors listing. Test the replacement of the drum from a recording traced by Gamelan. - Accurately identify which tracks to replay; substitute an identified track to another; replay the final mix session with the replaced tracks; identify contributors of the project.

Development results spread on several levels:

- an operational meta-environment with production tracking (Gamelan Tracker),

- a strongly-committed ontology for digital music production domain (DiMPO), 
- a raw trace interpreter (logs2dimpo),

- a timeline visualizer (Gamelan Viewer),

- a query management application (OwlimQueryManager), with a set of queries related to the use cases.

\subsection{Architecture Overview}

The technical goal of the Gamelan research project is to create a software "metaenvironment" (also called Gamelan), in the sense that it aims at producing knowledge over production environment utilization. It integrates some music production softwares and is able to describe the production workflow, from source to final product, at an abstraction level which is higher than the data level. For this purpose, methods from several fields were combined: trace engineering for the tracking system, and knowledge modeling and engineering for ontology design and the querying and visualizing system, as shown on Fig.1.

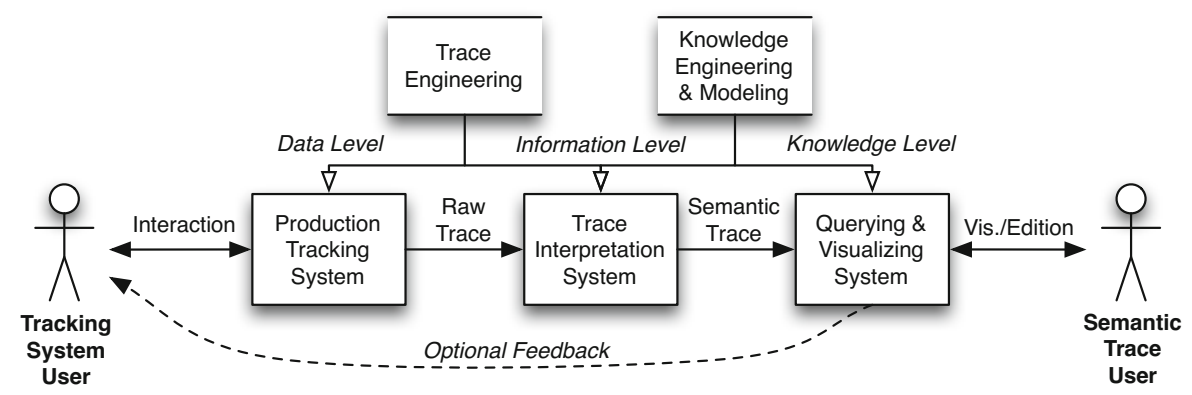

Fig. 1. Gamelan architecture overview

Gamelan promotes two categories of users: users of the tracking system, who generate traces while they interact with the digital tools of the studio during the production process, and users of these traces, at the other end of the whole system. If a user of the tracker is also user of the trace, then he or she simply get a feedback loop in the creative process, e.g. a live process evaluation.

With these two users, one can see the whole Gamelan architecture as a threetiered system 1 , made of three layers sequentially chained, corresponding to Ackoff's Data, Information and Knowledge levels [4].

The left-most region of Fig.1 1 represents the trace collecting part of Gamelan, starting from the production activity of the tracking system user. This tracking module should respect the noninvasive constraint against creativity as much as possible. It feeds the system with raw traces which are precious but too difficult to exploit under this primitive form.

\footnotetext{
${ }^{1}$ Not to be confused with "client-server three-tier architecture".
} 
After this raw trace point, an ontology becomes necessary for any further operation, as one leave the simple data level, providing a reference knowledge model. The DiMPO ontology, standing for "Digital music production ontology", has been elaborated during the project [5]. The main technical features of Gamelan meta-environment include at different levels: tracking, acquisition, ingestion, reasoning, requesting, browsing, file genealogy visualization, integrity and authority checking, and archiving. Besides, Gamelan relies on standard formats, such as: RDF2, OWL3, Sesame, SparQL5 and OSC6.

\section{Production Tracking System}

The first part of the meta-environment deals with raw traces of production, through automatic logging of user interaction events and contextual data asking. It involves the tracking system user, at data level, to collecting production traces.

We define trace as the recordings of computer-mediated activity from program execution events. In a digital music production context, traces are both user interaction event logs, from applications and operating system, and production artifacts themselves, typically imported or exported files. The underlying hypothesis of Gamelan on production process tracking is that the digital realm allows to track a production activity without disturbing it too much, which is often admitted7.

\subsection{Tracking System User Interface}

The first development result is the production tracking system, which combines the GamelanTracker software and "gamelanized" production softwares, mainly Audacity. The development affects three layers:

- software and file system events tracking, based on messaging;

- production file movement monitoring and back-up recording;

- manual entry information collecting, via ontology-compliant user interface.

This part integrates musical and sound production softwares and has its own non-invasive user interface: instead of the common pop-up windows, we designed an unobtrusive menu, accessible from a small "Track on/off" checkbox icon, as shown on Fig.2, top left.

${ }^{2}$ Resource Description Framework, a standard model for data interchange on the Web.

${ }^{3}$ Web Ontology Language, for authoring ontologies or knowledge bases.

${ }^{4}$ Sesame is a de-facto standard framework for processing RDF data.

5 SparQL Protocol and RDF Query Language.

${ }^{6}$ Open Sound Control is a content format for messaging among digital devices.

${ }^{7}$ For instance see Dyke's thesis [6]: "The tracing of computer-mediated activity is a special situation in that it is both possible to be very specific in what is traced, and to do so without modifying the environment in a disruptive way." 


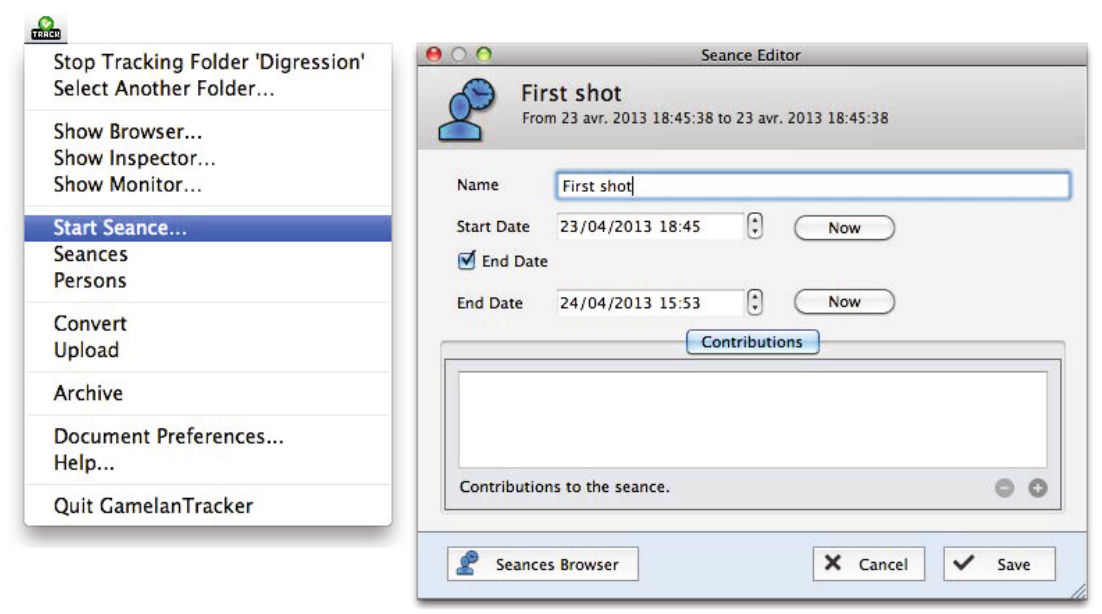

Fig. 2. GamelanTracker user interface (credit: INA)

\subsection{Software Activity Tracking Implementation}

Traces are to be mobilized in contexts that are never totally predictable and these inscriptions will report a reality that has evolved by itself. That is the reason why we designed a software activity tracking that is as agnostic as possible, through raw messaging, listening and logging.

The messaging part relies on an open-source standard commonly used in the computer music community: OSC (Open Sound Contro 8 ) developed at UC Berkeley [7, which is a communication protocol for modern networking technology, with a client/server architecture (UDP and TCP).

In order to produce usage data 89 , we modified open-source domain production softwares, mainly Audacity 9 , an open-source software for recording and editing sounds, written in $\mathrm{C}++$. We added specific OSC messaging functions into several functions of interest, such as open, save, save as, play, stop, cut, copy, paste, etc. This way, each time the user performs an action through a user-level function call of the production software, our modified software sends a complete OSC message, built with the following: application name, application version number, time stamp, function name, plus specific function parameters if needed.

Gamelan Tracker, a corresponding tracking application, has been developed. It receives and logs every message broadcasted during production time from three possible sources:

- "gamelanized" applications, for action logs (OSCMessages.txt)

- File System, for file movement logs (FolderState.txt)

- Operating System, for application change logs (CurrentApplication.txt)

8 http://opensoundcontrol.org

9 http://audacity.sourceforge.net 
2012-07-09 10:09:36546 +02 audacity 1.3 FileNew

2012-07-09 10:09:36553 +02 audacity 1.3 FileSaveAs test.aup

2012-07-09 10:09:36560 +02 audacity 1.3 ImportAudio test.aup noise.wav

2012-07-09 10:09:36561 +02 audacity 1.3 ImportAudio test.aup clicks.wav

2012-07-09 10:09:36563 +02 audacity 1.3 Select "noise", "clicks"; Begin="1.931"; End="10.014"

2012-07-09 10:09:36571 +02 audacity 1.3 ExportAudio test.aup mix.aif

2012-07-09 10:09:36581 +02 audacity 1.3 FileClosed test.aup

CurrentApplication. txt

2012-07-09 10:09:36544 +02 ApplicationActivated net.sourceforge.audacity

2012-07-09 10:09:36582 +02 ApplicationActivated com.apple.dt.Xcode

2012-07-09 10:09:36593 +02 ApplicationActivated com.apple.finder

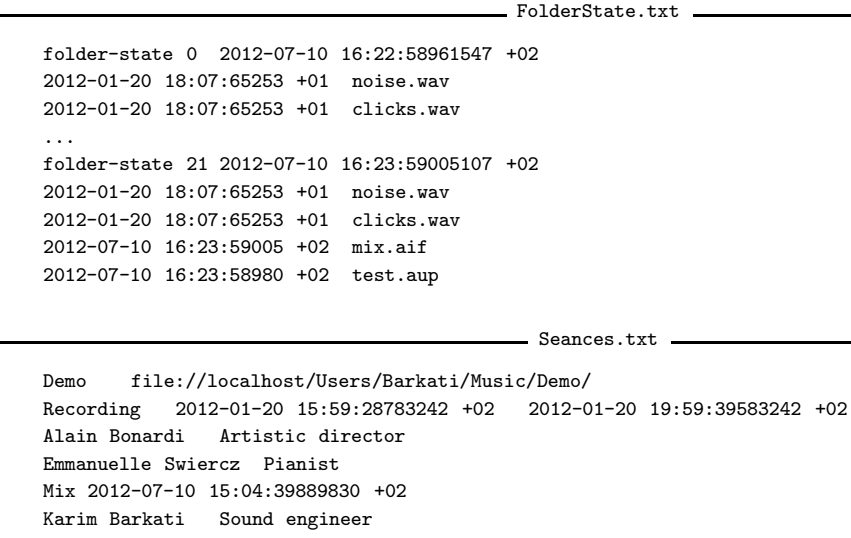

Fig. 3. Log files excerpts

GamelanTracker adds a reception time stamp and keeps track of every version of modified files in a backup folder for file genealogy analysis and preservation purposes. Fig. [3] shows excerpts of log files, reduced to fit in paper width (some timestamps and/or other information are truncated).

\subsection{Manual Informing Issues}

Software activity tracking is often not sufficient to fulfill our use cases. For instance, contributors listing (EMI use case) requires further contributor information that cannot be inferred from software traces. In these cases, at least some primary contextual information must be provided by a human operator, such as the user's name and the title of the work being produced (see Seances.txt excerpt on Fig.(3).

At first, production time seems to be the best time for asking the tracking system user to provide this information. But a design dilemma rapidly appears: on the one hand, the more contextual information feeds the system, the more informative the knowledge management can be, but on the other hand, the more a system asks the user to enter data while he or she is working, the more the user may reject the documenting system [10]. 
In our case, the balance between quantity and quality of information has to be adjusted in a close relationship with the ontology we have been incrementally developing with domain experts 11] and which is presented thereafter.

Temporal modalities have also to be anticipated in the information system, since the manual information can be entered during production time or temporally uncoupled, either by a producing user (e.g. a composer or a sound engineer) or by an external agent (e.g. a secretary or a curator).

\section{Trace Interpretation System}

This second part is hidden to both tracking system user and trace user. Nevertheless, it carries a crux task: interpret raw trace, to raise these traces from data level to information level. Firstly, a target representation language is required, calling for knowledge modeling. Secondly, an artificial interpretation program has to be designed and developed.

\subsection{Knowledge Modeling}

To formalize knowledge at stake, a domain ontology has been elaborated during the project, mainly by Antoine Vincent from UTC: DiMPO, standing for "Digital Music Production Ontology" [11. Modeling digital music production knowledge required to form an analysis corpus first, because of the lack of written documents. Then, the preservation aim leads to ensure the robustness of the model, which we addressed with a differential method.

Music Production Knowledge. Usually, the modeling phase begins with a corpus analysis from a collection of candidate-documents selected on their relevance [12. But in the case of digital music production, such a corpus does not exist, i.e. no written document can provide sufficient support to terms selection. Indeed, vocabulary, and consequently all the production process, relies on musical practices that are acquired more by experience than by teaching.

Thus, to achieve this essential phase of study, we needed to make up our own corpus, which is rather unusual in ontology making: several musical productions were followed to find out and adequately formalize invariants into an ontology.

With DiMPO, we do not seek to explain sound nor music (the what, as in MusicXML kind of languages) but the way it is produced (the how), i.e. a formal language for audio production process. This language is devoted to the representation of what we might call the "music production level", referring to the "knowledge level" of Allen Newell: we want to represent the work at the right abstraction level, neither too concrete because too technology dependent and therefore highly subjected to obsolescence, nor not concrete enough because information would be too vague to be usable [3].

Production Process Modeling. To create the DiMPO representation language and implement its operationalization, we applied the Archont 10 method of Bachimont [13. The modeling of digital music production followed three steps:

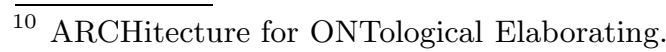


1. Normalization of the meanings of selected terms and classification in an ontological tree, specifying the relations of similarity between each concept and its parent concept and/or sibling concepts: we then have a differential ontology;

2. Formalization of knowledge, adding properties to concepts or constraining relation fields, to obtain an referential ontology;

3. Operationalization in the representation language, in the form of a computational ontology.

After a phase of collecting our corpus and selecting candidate terms, we took the first step in the form of a taxonomy of concepts, in which we strived to maintain a strong semantic commitment in supporting the principles of the differential semantics theory presented thereafter. This taxonomy has been performed iteratively, since it is tightly dependent on our participation in successive productions. Thus, at each new integration to the creation or the updating of a work, we flatten and question our taxonomy and term normalization, in order to verify that the semantic commitment is respected. For common features, we import standard ontologies, among which vCard 11 for standard identity information.

In order to ease incremental development and testing, we divided the ontology in two parts. The first part represents the model, with classes and properties, uploaded on a dedicated server icm.ircam.fr 12 . The second part contains modelcompliant data sets, with "DiMPO individuals", uploaded on an OWL server gsemantic.ircam.fr 13 (an OpenRDF Sesame repository with the OWLIM-Lite engine).

The Differential Approach. The differential approach for ontology elaboration systematically investigates the similarity and difference relations between each concept, its parent concept and its sibling concepts. So, while developing this structure, we tried to respect a strong ontological commitment by applying a semantic normalization, i.e. by asking, for each concept $c$, the four differential questions of Table 1 .

To carry out this semantic normalization task from a practical point of view, we used DOE 14 14 and Protég 15 softwares, for both taxonomy building, refining and exporting (RDFS, OWL, etc.). Fig.4 shows an excerpt from DiMPO

Table 1. The four differential questions

1. $c \sim$ parent $(c)$ - Why does this concept inherit from its parent concept?

2. $c \nsim$ parent $(c)$ - Why is this concept different from its parent concept?

3. $c \sim \operatorname{sibling}(c)$ - Why is this concept similar to its sibling concepts?

4. $c \nsim$ sibling $(c)$ - Why is this concept different from its sibling concepts?

\footnotetext{
11 http://www .w3.org/Submission/vcard-rdf

12 http://icm.ircam.fr/gamelan/ontology/2013/04/03/DiMP0.owl

13 http://gsemantic.ircam.fr

14 http://www.eurecom.fr/ troncy/DOE/ Differential Ontology Editor.

15 http://protege.stanford.edu/
} 


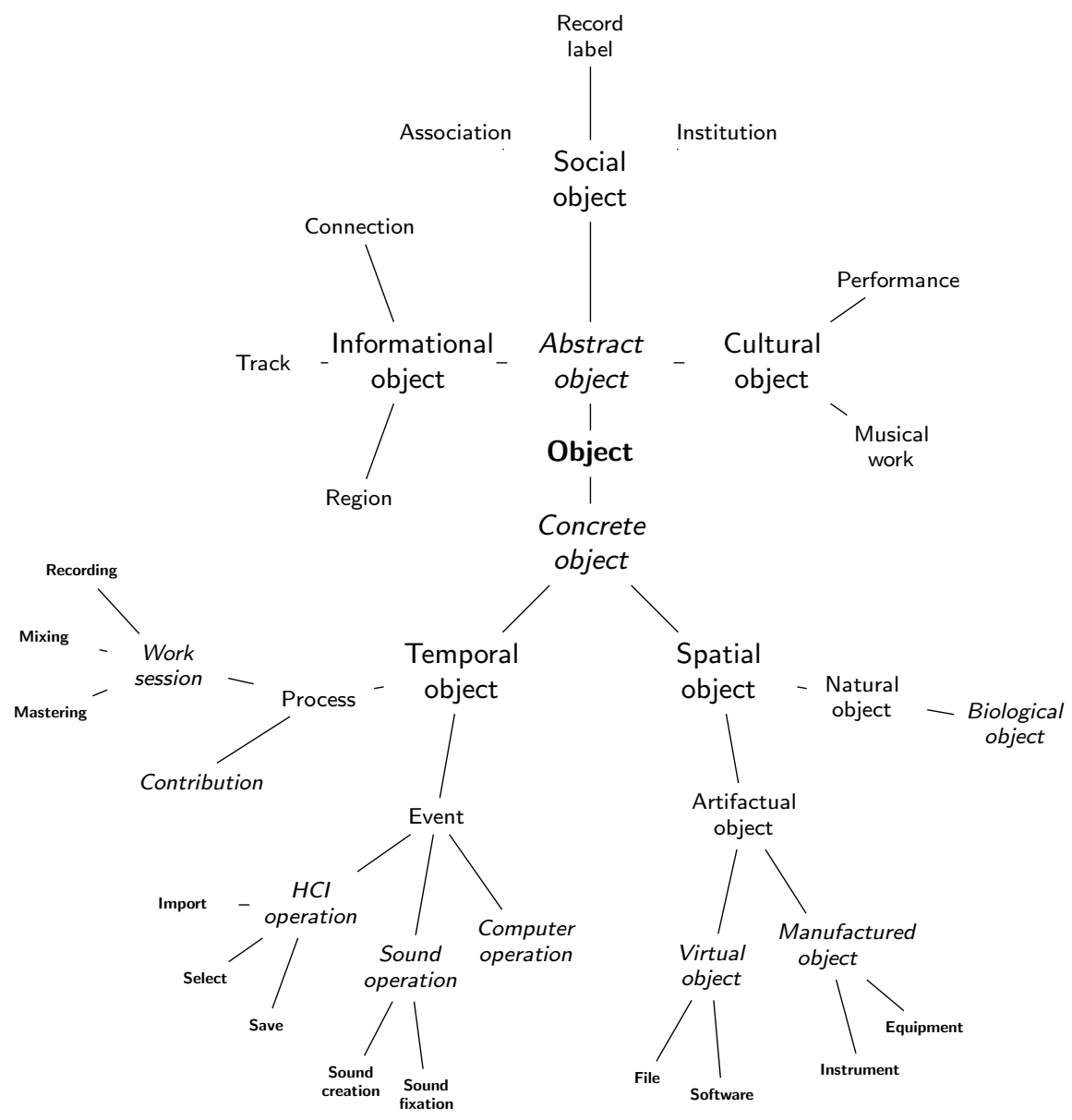

Fig. 4. Excerpt from the differential taxonomy (credit: UTC)

taxonomy. At the end of this recursive process, one obtains a domain-specific differential ontology, where the meaning of all terms have been normalized and that allows to develop the vocabulary needed for the next steps to reach the development of the representation language of the music production process.

As a result of the differential method, domain vocabulary mostly occurs in the leaves of such an ontological tree: work, performance, version; connection, graphical object, track, region; association, enterprise, institute; musical score, instrument, brass, strings, percussions, winds; effect box, synthesizer; file, session file, program; create, delete, edit; content import, content export; listen, play, work session, current selection; etc. A large set of properties completes this domain ontology.

\subsection{Artificial Interpretation}

Interpretation of raw traces according to the ontology yields "semantic traces" as interrelated ontological individuals, conformable to DiMPO. To perform this 
interpretation, we implemented the logs2dimpo.pl translation program in Perl language, which we chose for its text file parsing facilities. This program transforms raw logs into DiMPO individuals. Within Gamelan, this translator is called from Gamelan Tracker, and checks for uniqueness of each individual against a remote knowledge base when necessary (Fig. 5 ).

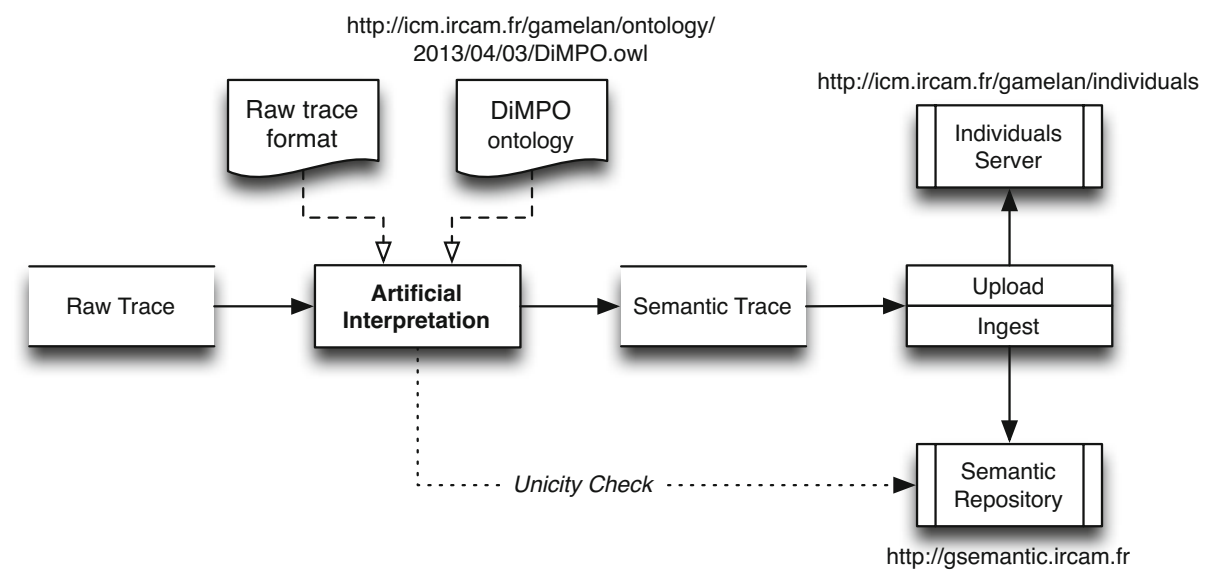

Fig. 5. Trace interpretation and management

Raw Traces Interpretation. Raw traces are not directly informative nor exploitable under this raw form of log files (see Fig. 3). The logs2dimpo program interprets theses traces according to DiMPO ontology and OWL language in order to convert them into "semantic traces", i.e. ontological individuals. A few interrelated DiMPO individuals are shown on Fig.6] as "owl:NamedIndividual" elements, identified by a unique URI that ensures relations between individuals.

Uniqueness Checking. If a DiMPO individual produced by the translator is intended to be ingested into an existing semantic repository, then the translator shall check whether this individual is already recorded, to ensure individual uniqueness. A mechanism of index attribution recovers current indexes for each DiMPO class present in the semantic repository before individual numbering.

Individual and Ontology Servers. As production management may involve several users, we designed additional online features. For instance, before being ingested into the semantic repository, semantic traces are uploaded in a server dedicated to DiMPO individual 16 , in order to provide individuals with an internet location. Moreover, another server has been dedicated to DiMPO ontology versions 17 .

\footnotetext{
16 http://icm.ircam.fr/gamelan/individuals

17 http://icm.ircam.fr/gamelan/ontology/2013/04/03/DiMPO.owl
} 


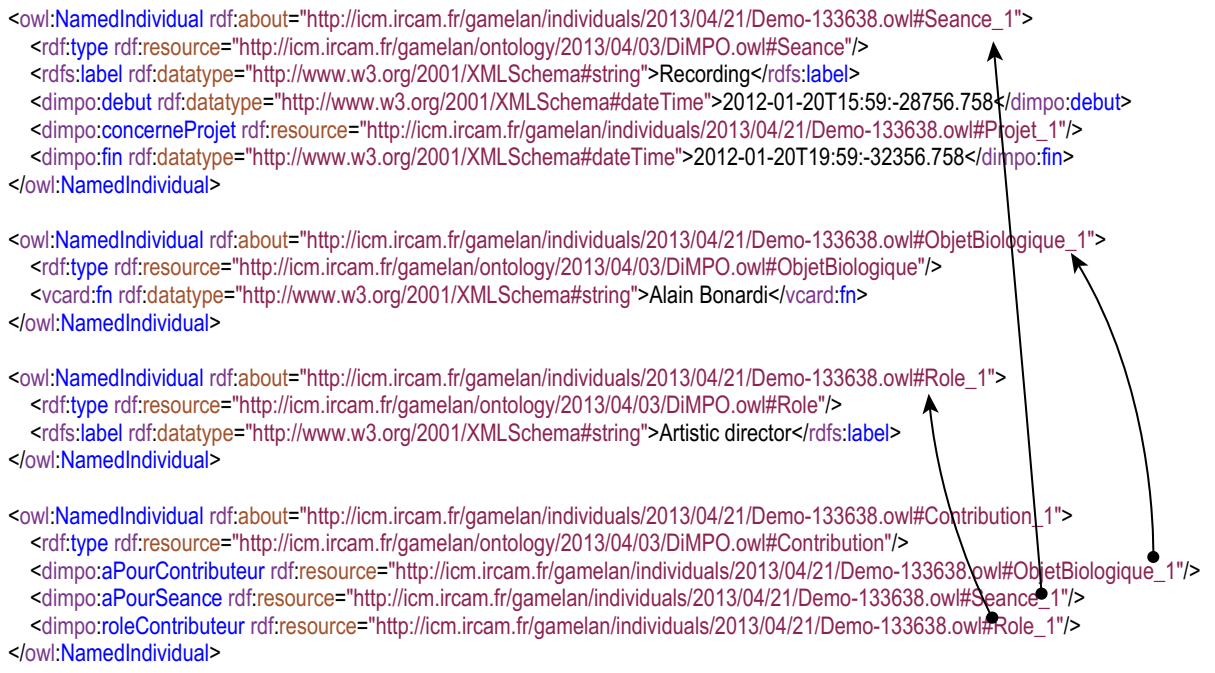

Fig. 6. Some interrelated DiMPO individuals

\section{Querying and Visualizing System}

This third part aims at bringing production knowledge to the trace user, through knowledge engineering techniques. It has spread on several operational tasks: manage a server for the "semantic traces", deploy a semantic repository with reasoning capabilities from the ontology, and prototype use case queries.

\subsection{Semantic Repository}

A Sesame OpenRDF semantic repository has been installed from an Ontotext OWLIM-Lite version. It handles structured data storage and management, reasoning and querying.

Data Storage and Management. OWL/RDF data ingestion on the semantic repository is triggered by a short Java program integrated into Gamelan Tracker and using Sesame API. An online graphical interface allows repositories management at http://gsemantic.ircam.fr.

Reasoning. The OWLIM inference engine performs completion of facts through "total materialization" at load time. This reasoning strategy slows down upload but speeds up retrieval and querying, which is what we have chosen.

Querying. The semantic repository embeds a query engine accessible through HTTP. We use the SPARQL 18 language to write RDF queries against the semantic repository, with triple patterns syntax.

$\overline{18 \text { SPARQL is }}$ an RDF query language, appeared in 2008 and well-suited for triples. Its recursive acronym stands for "SPARQL Protocol and RDF Query Language". 


\subsection{Triplestore Querying}

The digital archival issue of provenance should be avoided or at least diminished upstream from the ingest step. The Gamelan meta-environment allows to detect crucial missing information by reasoning on the combination of software traces and user information, from expert knowledge. These features, important to the trace user, are partially carried out through production tracking and common knowledge management tools, such as domain ontology, query engine, and semantic repository. For example, one can query the semantic repository in order to check whether expected contributors and their roles on the project are well informed or not (EMI use case, results on Fig.7).

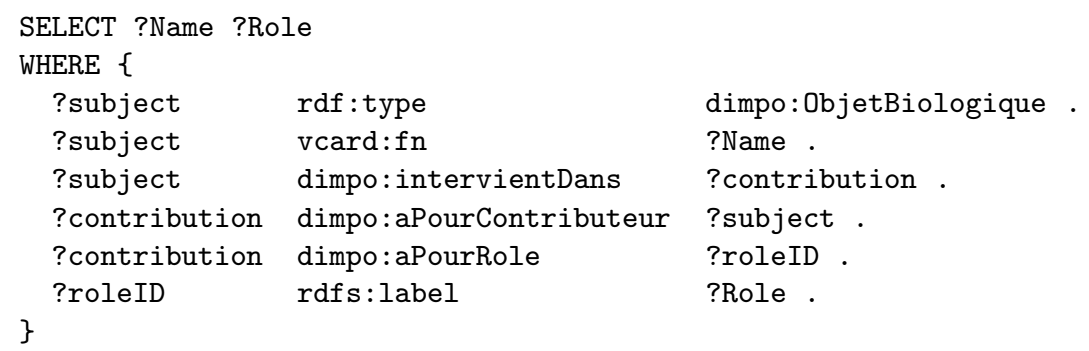

\begin{tabular}{|l|l|}
\hline Name & Role \\
\hline "Tristan Leblanc"^^^xsd:string & "Développeur"^^xsd:string \\
\hline "Laurent Vinet"^^^xsd:string & "Beta-testeur"^^^xsd:string \\
\hline
\end{tabular}

Fig. 7. Contributors checking

A query storage and management application has been developed to capitalize on and manage trace user queries: sets of queries are designed and managed in OwlimQueryManager, a user-friendly application developed on purpose, as shown on Fig.8.

\subsection{Time Axis Reconstruction}

As we are dealing with production workflows, time axis reconstruction is essential to trace user understanding.

However, archiving music and sound production is generally limited to the rudimentary archiving of a final version (called "master version"), at the end of the production process. Whereafter it is clearly impossible to trace the production history from this single object, nor to take back and modify the process in a different perspective, as needed in repurposing EMI use case for instance. This led us to ensure strong timing properties through our trace-based system, not only time stamping user events from the production tools when emitting messages, but also independently time stamping a second time these events in the logging module when receiving messages. This allows to reconstruct the time axis of the production safely. 


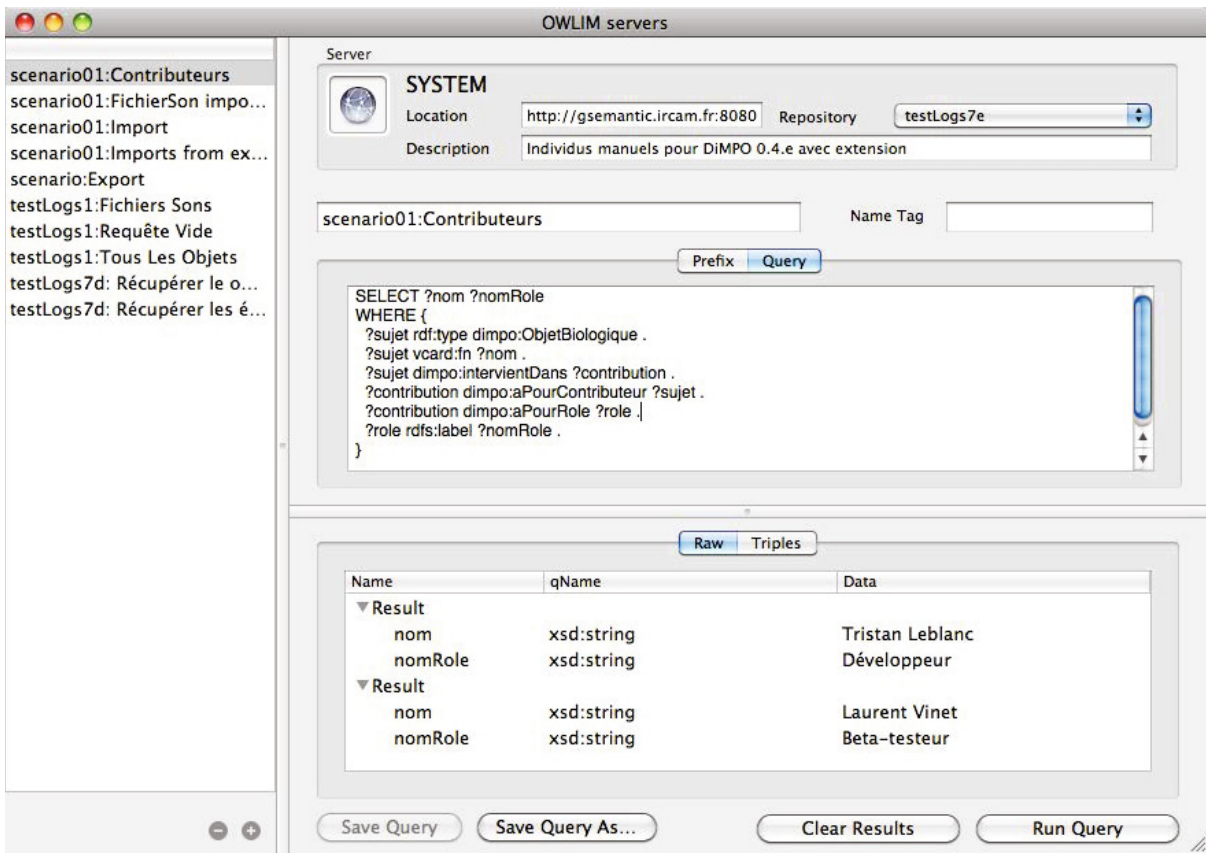

Fig. 8. OwlimQueryManager user interface (credit: INA)

For example, a typical query can retrieve and chronologically order audio files movements (imports and exports) in a musical project (results on Fig.91).

\subsection{Timeline Visualization}

Furthermore, the GamelanViewer timeline visualization tool shown in Fig.10, can draw a global view to help query results understanding, typically showing the genealogy of the files used during the production. For example, Gamelan can infer which files were used to compose a mixed file, hierarchically, and also deduce which is the "last mix" in a set of file; this kind of knowledge is of prime importance when a composer or a producer decides to remix a work years later, as pointed out in INA/GRM use case.

\subsection{Production Patterns}

When DiMPO ontology reached a decent and stabilized level, we entered a second phase of the ontological research: production patterns design. Production patterns define audio creation acts, such as editing, shown on Fig.11 (in UML). The use of these patterns allows to represent a set of actions with a musical meaning, incorporating the vocabulary developed in the ontology. 


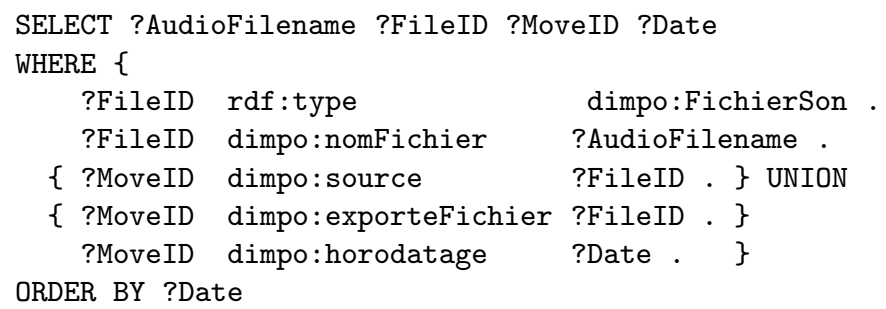

\begin{tabular}{|l|l|l|l|}
\hline AudioFilename & FilelD & MovementID & Date \\
\hline "nuagesGris extrait1 mono.wav"^^xsd:string & scenario01:FichierSon 1 & scenarioo1:ImportAudio 1 & 2013-04-05T12:20:52.000 \\
\hline "nuagesGris extrait2 mono.wav"^^xsd:string & scenario01:FichierSon 2 & scenario01:ImportAudio 2 & 2013-04-05T12:20:55.000 \\
\hline "montage1.wav"^^xsd:string & $\underline{\text { scenario01:FichierSon_3 }}$ & $\underline{\text { scenarioo1:ExportAudio 1 }}$ & $\underline{\text { 2013-04-05T12:22:14.000 }}$ \\
\hline
\end{tabular}

Fig. 9. Retrieval and ordering of timestamped file movements

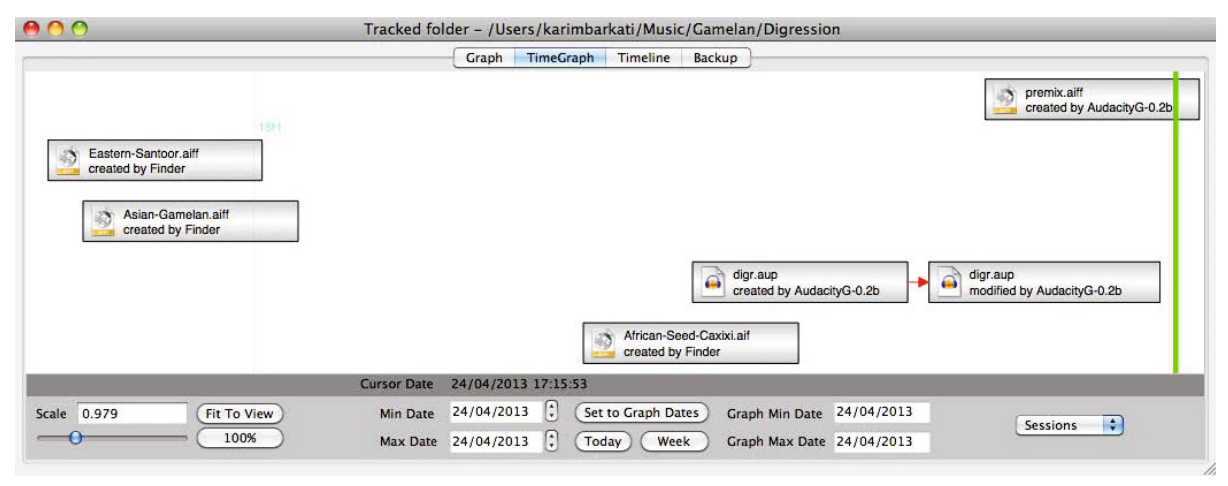

Fig. 10. Timeline visualization (credit: INA)

Our query patterns are grounded on these production patterns. Reuse of ontology vocabulary in production patterns eases their translation into query patterns, especially when using RDF-compliant query languages such as SPARQL, as we do onto our Sesame repository containing OWL individuals.

Here, knowledge can be viewed as bilocalized: on the semantic repository side for interrelated individuals of the semantic trace database, and on the query manager side for the formalized relations of the query patterns base. The more accurate production patterns are, the more useful derived query patterns can be, and the less the trace user has to know or to learn about the details of both the query language and the ontology.

\section{Discussion}

In this section, we discuss modeling, knowledge support, and time horizons of production process tracing. 


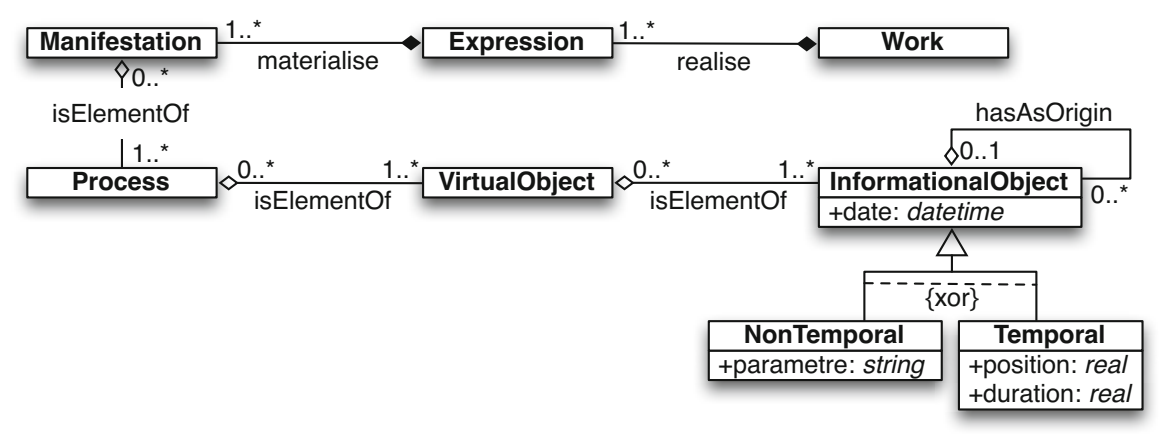

Fig. 11. A production pattern diagram for editing (credit: UTC)

\subsection{Model Pervasiveness and Design Heuristics}

As suggested in the architecture overview section (Sec.1.3), apart from the operational tracking that has to remain agnostic, the domain ontology drives most functional modules of our system at each level:

Data - the semi-automatic collecting module, i.e. software activity tracking and manual informing design;

Information - the translation module that interprets raw data (both automatic usage data and manual user data) according to the ontology;

Knowledge - the semantic engine reasoning on the preprocessed information, and answering requests;

Understanding - the query manager module for data browsing, and the viewer module that provides graphical representations, such as timelines and file genealogy trees.

According to Ackoff's model 4], knowledge management depends on the ability to transform data and information into knowledge, a task where ontologies proved key tools [1516], thanks to their semantic capabilities. That led us to undertake our semantic research in professional music knowledge modeling.

Yet, despite their power and thus their pervasiveness, ontologies remain human artifacts never elaborated without design heuristics. We developed a stronglycommitted ontology incrementally, dipping into music productions with domain experts and submitting ontology drafts to them. This incremental approach continued during the next phases: during software development - with developers feedback -, and during tests and validation - with user groups feedback.

The differential approach we applied along the ontology development cycles balances the random part brought by heuristics but cannot eliminate it in any way. Ontology-driven knowledge management should be aware of this contingency dimension. 


\subsection{A Knowledge Support Language}

The descriptive approach is not about keeping the content stored, because content is usually partial, incomplete or poorly defined (closed formats, imperfect knowledge of it, etc.). Rather, it is better to retain a description of the content that enables to reproduce it. The description may include the main points to reproduce, the author's intention to comply with [17, the graphical appearance, and any potentially informative element to the reader for reproduction.

So, the description of the content of a work is an approach increasingly adopted in response to the technical complexity (mostly digital) of content: instead of maintaining a technical object that we may no longer know how to reuse, we shall aim at constructing a description that allows to recreate this object with the tools we will have at hand when the time comes. Such a description necessarily introduces a deviation from the original. So the challenge is to minimize the impact of this difference on the integrity and authenticity of the work.

The main question is how to determine such a description language. The music score used in the so-called classical music, is a good example of such a description language. Instead of stepping on the impossible task to keep a musical object before recording techniques, musicians preferred to keep the instructions to create $i t$. Now, the complexity of the works, the mutability and fragility of digital systems and objects imply that it is impossible to guarantee that a technical object will still be executable, readable or editable in the future.

Several approaches are possible, but some semiotic and logic work is necessary to identify such a description stage:

- Semiotic, because it is necessary to characterize the objects mobilized in a production, define their significance and propose an associated representation;

- Logical, since this representation must be enrolled in a language for operationalization in the proposed meta-environment.

The combination of these semiotic and logic approaches are key concepts to unlock knowledge possibilities of both the work as an artifact and the creation as a process.

\subsection{Horizons of Production Process Tracing}

As far as we know, music production process tracking has never been done yet, except for some isolated generative music programming under version control system (like cvs, svn or git). Regarding tracking, we distinguish between user data and usage data; the former corresponds to the manual informing data and the latter to the automatic tracking data.

As a meta-environment, Gamelan traces data during the production activity and utilizes formalized knowledge to exploit collected data, both during and after production time. This production tracing strategy aims for several beneficiaries and time horizons: 
In the immediate time of production - The composer, audio producer, may turn back their own work during the production, to explore various options or correct the undesirable consequences. Use cases show needs for a selective offline "undo" instruction, to cancel a specific operation afterwards, even when the production software was quit. There is also, for the composer or the sound engineer, an opportunity to see and understand the overall work progress of composition or production during the process itself.

In the intermediate time of collection - The composer, or the institution that manages art works, may return on a given work to recreate it or reuse some parts of the content of the final work, which are usually no longer accessible.

In the long term preservation - The work becomes a memory and a relic, the challenge is to preserve the artistic and technical information to understand, interpret and re-perform, as in the case of contemporary works using real-time programs on stage.

\section{Conclusions}

Traditional places of creation generate final artifacts or art works that are closed: creativity is emphasized but the creation process is most often lost, locking both artifact structure recovery and process understanding. In this context, living labs often attempt to trace the creation process, by recording actions for usage study. But, be they artifacts or process recordings, how to understand digital studio outputs?

\subsection{From Production Traces to Production Knowledge}

We presented how we combined a trace-based architecture and an ontologydriven knowledge management system, the latter being built upon differential semantics theory for sustainability, in order to raise production activity traces from data level to information level, then to knowledge level. Technically, semiautomatic production tracking feeds an interpretation program which, in turn, feeds a semantic repository. The idea of such a production meta-environment, viewed as a trace-based system, meets clear needs in the community. As of now, Gamelan addresses intermediate artifacts preservation, file genealogy visualization, and contributors' identification. Moreover, our ontological work already points to the solution of various scientific challenges:

- Representation language for managing the production process;

- Description language for representing the content of a work, with the diversity of its components;

- Integration of both languages in a single control environment. 


\subsection{From Production Knowledge to Production Understanding}

Digital studios and living labs produce a great amount of traces [18] that could be better understood - and thus more easily exploitable - using semantic trace strategies such as those developed within Gamelan for the case of digital music production, combining semi-automatic activity tracking with content and process modeling.

We presented how a knowledge management approach for digital music production workflows could be set up. This trace-based system already showed improving primary understanding, especially through visualizing interfaces. As we stated, further understanding would be of great utility at several time horizons: in the immediate time of production, in the intermediate time of collection, and in the long term of preservation.

Currently, our system can support trace interpretation only up to a certain point, which is style [19|20]. Meeting style understanding would need further modeling effort at higher level, which should be partially eased by our production patterns and the trace collecting and interpreting methods we developed. Further studies shall evaluate to what extent creation process style can be modeled.

Envision style modeling from semantic traces will require to rely on experts of art humanities at least, typically in our music production case on musicologists and composers.

Of course, approaching style understanding is of great interest [21 22]. Nevertheless, it may be perceived by creators as a provocative attempt to unraveling the mystery of art and creation. Then, we are entitled to wonder if art objects opacity regarding their making is not a consequence of a mystery will from creators. If it is the case, new understanding capabilities could be perceived both as a cure and a poison.

This is probably a first class concern of future Digital Humanities culture 2324. From our point of view, the advent of style pattern understanding would not reduce creative processes nor creativity potentials though, but rather most likely shift them.

Acknowledgments. The Gamelan project is funded by French National Research Agency. It started in 2009 and will end in 2013. The partners are:

- IRCAM (Institut de Recherche et Coordination Acoustique/ Musique),

- Heudiasyc - UMR 7253 CNRS UTC,

- INA (Institut National de l'Audiovisuel),

- EMI Music France.

\section{References}

1. Laflaquière, J., Settouti, L.S., Prié, Y., Mille, A.: Trace-based framework for experience management and engineering. In: Gabrys, B., Howlett, R.J., Jain, L.C. (eds.) KES 2006. LNCS (LNAI), vol. 4251, pp. 1171-1178. Springer, Heidelberg (2006)

2. Georgeon, O., Henning, M.J., Bellet, T., Mille, A.: Creating cognitive models from activity analysis: A knowledge engineering approach to car driver modeling. In: International Conference on Cognitive Modeling, pp. 43-48 (2007) 
3. Newell, A.: The knowledge level. Artificial Intelligence 18(1) (1982)

4. Ackoff, R.: From data to wisdom. Journal of Applied Systems Analysis 16(1), 3-9 (1989)

5. Vincent, A., Bonardi, A., Bachimont, B.: Étude des processus compositionnels: Un langage pour représenter les processus de production sonore (2013)

6. Dyke, G.: A model for managing and capitalizing on the analyses of traces of activity in collaborative interaction. PhD thesis, ENS Mines (2009)

7. Wright, M., Freed, A., Momeni, A.: Opensound control: state of the art 2003. In: Proceedings of the 2003 Conference on New Interfaces for Musical Expression, National University of Singapore, pp. 153-160 (2003)

8. McLeod, I., Evans, H., Gray, P., Mancy, R.: Instrumenting bytecode for the production of usage data. In: Computer-aided Design of User Interfaces IV, pp. 185-195 (2005)

9. Smith, S., Schank, E., Tyler, B.: Instrumented application for transaction tracing, US Patent App. 11/092,428 (March 29, 2005)

10. Barki, H., Hartwick, J.: Measuring user participation, user involvement, and user attitude. MIS Quarterly, 59-82 (1994)

11. Vincent, A., Bachimont, B., Bonardi, A., et al.: Modéliser les processus de création de la musique avec dispositif numérique: représenter pour rejouer et préserver les œuvres contemporaines. Actes des Journées Francophones d'Ingénierie des Connaissances (2012)

12. Rousseaux, F., Bonardi, A.: Parcourir et constituer nos collections numériques. In: CIDE Proceedings, pp. 133-142 (2007)

13. Bachimont, B.: Ingénierie des connaissances. Hermes Lavoisier, Paris (2007)

14. Bachimont, B., Isaac, A., Troncy, R.: Semantic commitment for designing ontologies: A proposal. In: Gómez-Pérez, A., Benjamins, V.R. (eds.) EKAW 2002. LNCS (LNAI), vol. 2473, pp. 114-121. Springer, Heidelberg (2002)

15. Gruber, T.: Toward principles for the design of ontologies used for knowledge sharing. International Journal of Human Computer Studies 43(5), 907-928 (1995)

16. Fensel, D., Van Harmelen, F., Klein, M., Akkermans, H., Broekstra, J., Fluit, C., van der Meer, J., Schnurr, H., Studer, R., Hughes, J., et al.: On-to-knowledge: Ontology-based tools for knowledge management. In: Proceedings of the eBusiness and eWork, pp. 18-20 (2000)

17. Gaillard, L., Nanard, J., Bachimont, B., Chamming's, L.: Intentions based authoring process from audiovisual resources. In: Proceedings of the 2007 International Workshop on Semantically Aware Document Processing and Indexing, pp. 21-30. ACM (2007)

18. Følstad, A.: Living labs for innovation and development of information and communication technology: a literature review. The Electronic Journal for Virtual Organizations and Networks 10(7), 99-131 (2008)

19. Pachet, F.: The future of content is in ourselves. Computers in Entertainment (CIE) 6(3), 31 (2008)

20. Bonnardel, N., Zenasni, F.: The impact of technology on creativity in design: An enhancement? Creativity and Innovation Management 19(2), 180-191 (2010)

21. Carney, J.D.: The style theory of art. Pacific Philosophical Quarterly 72(4), 272$289(1991)$

22. Dubnov, S., Assayag, G., Lartillot, O., Bejerano, G.: Using machine-learning methods for musical style modeling. Computer 36(10), 73-80 (2003)

23. Giraud, F., Jauréguiberry, F., Proulx, S., et al.: Usages et enjeux des technologies de communication. Liens Socio (1970)

24. Wang, F.Y.: Is culture computable? IEEE Intelligent Systems 24(2), 2-3 (2009) 